\title{
Erratum: Torque correlation length and stochastic twist dynamics of DNA [Phys. Rev. E 89, 062706 (2014)]
}

Edward J. Banigan and John F. Marko

(Received 1 April 2016; published 20 April 2016)

DOI: 10.1103/PhysRevE.93.049904

There is an erroneous factor of 2 in Eq. (2). The analysis and conclusions of the paper are unaltered, but the factor alters the following equations.

Equation (2) should read

$$
\zeta \dot{\theta}(s)=-\frac{\delta E}{\delta \theta}+f(s, t)=\frac{1}{\beta}\left(c_{1} \partial_{s}^{2} \theta-c_{2} \partial_{s}^{4} \theta\right)+f(s, t) .
$$

Equations (3), (4), (7), (11), and (12) are corrected simply by replacing factors of $\beta$ with $\beta / 2$. For example, Eqs. (3) should read

$$
\begin{aligned}
\left.\theta\right|_{s=0} & =\omega_{0} t, \\
\left.c_{2} \partial_{s}^{2} \theta\right|_{s=0} & =\left.c_{2} \partial_{s}^{2} \theta\right|_{s=L}=0, \\
\left.\zeta_{B} \dot{\theta}\right|_{s=L} & =-\left.\frac{1}{\beta}\left[c_{1} \partial_{s} \theta-c_{2} \partial_{s}^{3} \theta\right]\right|_{s=L} .
\end{aligned}
$$

Next, Eq. (14) should read

$$
\beta \zeta \partial_{t} \theta=c_{1} \partial_{s}^{2} \theta-c_{2} \partial_{s}^{4} \theta+f(t)
$$

Equation (15) should read

$$
\begin{aligned}
\beta \zeta_{\perp} \partial_{t} R= & i\left[\left(c_{2} \partial_{s}^{4} \theta-c_{1} \partial_{s}^{2} \theta\right) \partial_{s}^{2} R+\left(c_{2} \partial_{s}^{3} \theta-c_{1} \partial_{s} \theta\right) \partial_{s}^{3} R\right] \\
& -A \partial_{s}^{4} R+\beta F \partial_{s}^{2} R+g_{x}(t)+i g_{y}(t) .
\end{aligned}
$$

Equation (16) should read

$$
R_{q}(\omega)= \begin{cases}\frac{g_{q}(\omega)}{A q^{4}+\tau q^{3}+\beta F q^{2}-i \beta \zeta_{\perp} \omega} \quad \text { for equilibrium experiments [Fig. 1(a)] } \\ \frac{g_{q}(\omega)}{A q^{4}+\beta\left[\zeta_{B}+\zeta\left(L-s_{0}\right)\right] \omega_{0} q^{3}+\beta\left(F-i \zeta \omega_{0}\right) q^{2}-i \beta \zeta_{\perp} \omega} & \text { for nonequilibrium experiments [Fig. 1(b)]. }\end{cases}
$$

Similarly, the relaxation times $T_{k}$ given on page 2 are $T_{k}=\frac{\beta \zeta}{\lambda_{k}}$ for all $k$. This correction also applies to the relaxation and crossover times in the second column of page 3 .

In the Appendixes, the following equations are corrected. Equation (C1) should read

$$
\begin{aligned}
C_{\tau}(s, s, t) & =\frac{2}{L} \int_{0}^{\infty} \frac{\exp \left\{\frac{-\left(\frac{k \pi}{L}\right)^{2}\left(c_{1}+\left(\frac{k \pi}{L}\right)^{2} c_{2}\right) t}{\beta \zeta}\right\}}{c_{1}+c_{2}\left(\frac{k \pi}{L}\right)^{2}} d k \\
& \approx \frac{2}{L}\left(\int_{0}^{L / \xi \pi} \frac{\exp \left(\frac{-c_{1} \pi^{2}}{\beta \zeta L^{2}} k^{2} t\right)}{c_{1}} d k+\int_{L / \xi \pi}^{\infty} \frac{\exp \left(\frac{-c_{2} \pi^{4}}{\beta \zeta L^{4}} k^{4} t\right)}{c_{2}\left(\frac{k \pi}{L}\right)^{2}} d k\right) .
\end{aligned}
$$

The corresponding relaxation times mentioned in Appendix $\mathrm{C}$ are corrected as above.

Equation (D1) should read

$$
\beta \partial_{t} \Omega=\frac{1}{\zeta}\left(c_{1} \partial_{s}^{2} \Omega-c_{2} \partial_{s}^{4} \Omega\right)+\frac{1}{\zeta_{\perp}}\left(\partial_{s} \vec{r} \times \partial_{s}^{2} \vec{r}\right) \partial_{s} \partial_{t} \vec{r}+f(t)
$$

Equation (D2) should read

$$
\begin{aligned}
\beta\left[\zeta_{\|} \hat{t} \hat{t}+\zeta_{\perp}(\mathbf{I}-\hat{t} \hat{t})\right] \partial_{t} \vec{r}= & \partial_{s}\left[\left(c_{1} \Omega-c_{2} \partial_{s}^{2} \Omega\right)\left(\partial_{s} \vec{r}\right) \times \partial_{s}^{2} \vec{r}\right] \\
& -A \partial_{s}^{4} \vec{r}+\beta \partial_{s}\left(\Lambda(s) \partial_{s} \vec{r}\right)+\vec{g}(t) .
\end{aligned}
$$


Equation (D3) should read

$$
\begin{aligned}
\zeta_{\perp} \partial_{t} R= & \left(F-i \zeta \omega_{0}\right) \partial_{s}^{2} R+i\left[\zeta_{B}+\zeta\left(L-s_{0}\right)\right] \omega_{0} \partial_{s}^{3} R \\
& -\frac{A}{\beta} \partial_{s}^{4} R+g(t)-i \zeta \omega_{0} \Delta s \partial_{s}^{3} R .
\end{aligned}
$$

Equations (D4) and (D5) are corrected by changing factors of $\zeta, \zeta_{B}$, and $\zeta_{\perp}$ into factors of $\zeta / 2, \zeta_{B} / 2$, and $\zeta_{\perp} / 2$, respectively.

Finally, the locally determined torque given at the bottom of the first column of page $6 \operatorname{should} \operatorname{read} \tau\left(s_{0}\right)=\beta\left[\zeta_{B}+\right.$ $\left.\zeta\left(L-s_{0}\right)\right] \omega_{0}$. 